\title{
Identification of microRNAs in ecological model plant Mimulus
}

\author{
Muhammad Younas Khan Barozai ${ }^{\star}$, Muhammad Din, Iftikhar Ahmed Baloch
}

Department of Botany, University of Baluchistan, Quetta, Pakistan; *Corresponding Author: barozaikhan@gmail.com

Received 15 March 2011; revised 20 April 2011; accepted 7 May 2011.

\begin{abstract}
MicroRNAs (miRNAs) are small, non-coding and regulatory RNAs about 20 to 24 nucleotides long. Their conserved nature among the various organisms makes them a good source of new miRNAs discovery by comparative genomics approach. This study resulted in 34 miRNAs belonging to 21 families in ecological model plant Mimulus. All the miRNA families (mir 156, $157,159,160,164,172,319,393,395,397,399$, $400,403,417,419,472,782,854,858,867$ and 2112) are found for the first time in Mimulus. The MIR-399 is found as precursor miRNA cluster with 4 mature sequences. All 34 miRNA precursors form stable minimum free energy stem loop structure as their orthologues form and the mature miRNAs reside in the stem portion of the stem loop structure. Twenty eight are from Mimulus guttatus and six miRNAs belong to Mimulus lewisii. Their targets consist of dihydroflavonol-4-reductase, cycloidea-like protein, DNA-directed RNA polymerase II, maturase (matR) and transcription factors like; squamosapromoter binding, MYB, palmate-like pentafoliata 1.
\end{abstract}

Keywords: Comparative Genomics; MicroRNAs; Mimulus

\section{INTRODUCTION}

Mimulus is a diverse plant genus. It is also called as the monkey-flowers and musk-flowers. It belongs to the family Phrymaceae consist of about 150 species [1]. The Mimulus properties make this group of angiosperm plants ideal for ecological and evolutionary genomics research [2]. MicroRNAs (miRNAs) are non-coding, endogenous, small RNAs about 20 - 24 nucleotide long [3]. They are conserved in plants and animals [4,5] and play vital role in post transcriptional gene regulation
[6,7]. Primary transcript of mature miRNAs (pri- miRNAs) folds into a stable hair-pin/stem-loop structure forming miRNAs precursor (pre-miRNAs). The loop of pre-miRNA is detached to create a short double-stranded RNA (dsRNA), a single strand of the dsRNA acts as mature miRNA [8]. A special RNaseIII-like endonuclease, Dicer-like enzyme (DCL) in plants involved to process the mature miRNA production [9], that also mostly integrate the mature miRNA into the RNA induced silencing complex (RISC) [8]. The RISC complex negatively regulates gene expression either by inhibiting translation elongation or by triggering messenger RNA (mRNA) destruction on the basis of the degree of complimentary of miRNA within its target [10,11]. The miRNAs execute multipurpose functions in plant and animals like; in growth [12] organogenesis [12,13], transgene suppression [14], signaling pathway [15], environmental stresses [16,17], disease development [18] and defense against the invading viruses [19].

Mostly miRNAs are conserved among animals and plants $[5,20]$. The conserved nature of these miRNAs becomes a logical approach for identification of new orthologues by comparative genomics in other species [21,22].

Here we report thirty four new miRNAs, belonging to twenty one miRNA families by homology search from the known Mimulus ESTs. All the miRNA families (mir 156, 157, 159, 160, 164, 172, 319, 393, 395, 397, 399, 400, 403, 417, 419, 472, 782, 854, 858, 867 and 2112) are reported for the first time in Mimulus. All 34 miRNA precursors form stable minimum free energy (mfe) stem loop structures, as their orthologues form and the mature miRNAs reside in the stem portion of the stem loop structure. Twenty eight are from Mimulus guttatus and six miRNAs belong to Mimulus lewisii.

\section{MATERIALS AND METHODS}

\subsection{Identification of Candidate Sequences}

Almost same approach as we reported earlier [21], 
was used. The plant pre-miRNAs from the microRNA Registry Database (Version Rfam 16.0 released Sept 2010) [23], were BLAST against publicly available $\mathrm{Mi}$ mulus ESTs at http://blast.ncbi.nlm.nih.gov/Blast.cgi using blastn [24]. Adjusted blast parameter settings were as follows: expect values were set at 1000; low complexity was chosen as the sequence filter, database (others. Mimulus) program selection (somewhat similar sequence) and all other parameters were used as default. The candidate sequences FASTA formats with a range of 0 - 4 mismatches with the mature sequences were saved and single tone EST was created for each miRNA after removing the repeated ESTs of the same gene.

To validate the initial candidate Mimulus miRNAs as non-protein coding, their sequences were subjected for protein homology search against protein database at NCBI using Blastx with default parameter [25].

\subsection{Creation of Hairpin Structures}

Zuker folding algorithm, MFOLD (version 3.2) [26], publicly available at http://www.bioinfo.rpi.edu/applica tions/mfold/rna/form1.cgi., was used to create the hairpin structure of the candidate's sequences. The parameters were adjusted same as reported earlier [21]. The stem portion of the hairpin checked for the mature sequences with at least 12 base pairs involved in Watson-Crick or $\mathrm{G} / \mathrm{U}$ base pairing between the mature miRNA and the opposite strand (miRNA*).

\subsection{Sequence and Structural Features Filtration}

To validate the miRNAs through the sequence and structural features filter, the GC content, Core mfe, hairpin mfe and Ch_ratio were calculated as described by Li et al., (2006) [27] with a little modification for Core mfe calculation, as described by Barozai et al., (2008) [21]. The mfe for core and hairpin structures were calculated by MFOLD (version3.2) [26] publicly available at http://www.bioinfo.rpi.edu/applications/mfold/ rna/form1.cgi. The parameters were adjusted same as described earlier. For Ch_ratio calculation, we divided the core mfe by the hairpin mfe, and the quotient is referred to as the ch_ratio.

\subsection{Conservation and Phylogenetic analysis of Mimulus miRNAs}

The Mimulus miRNA family; mir-156 conservation and phylogenetic analysis with Vitis vinifera, Arabidopsis thaliana, Oryza sativa and Populus trichocarpa orthologues was done with the help of publically available weblogo: a sequence logo generator [28] and ClustalW to generate cladogram tree using neighbor joining clus- tering method [29] respectively. The results were saved.

\subsection{Prediction of Mimulus miRNA Targets}

We predicted the Mimulus miRNA targets using the NCBI Blastn program [24]. The mRNA sequences showing $75 \%$ query coverage were selected and subjected to RNA-hybrid, a miRNA target prediction tool [30] for the confirmation of the targets. The results were saved.

\section{RESULT AND DISCUSSION}

\subsection{The Novel Mimulus miRNAs}

Total thirty four new Mimulus pre-miRNAs were identified after filtration and completion of the process. The thirty four potential Mimulus miRNAs belong to twenty one families (mir 156, 157, 159, 160, 164, 172, 319, 393, 395, 397, 399, 400, 403, 417, 419, 472, 782, 854, 858, 867 and 2112). Twenty eight are from Mimulus guttatus and six miRNAs belong to Mimulus lewisii. All the thirty four novel Mimulus miRNAs considered as a valid candidate after fulfilling the empirical formula for biogenesis and expression of the miRNAs, suggested by Ambros et al., (2003) [31]. The thirty four novel Mimulus pre-miRNAs fulfilled the criteria B, C and D. According to Ambros et al., (2003) [31] only the criterion $\mathrm{D}$ is enough for homologous sequences to validate as new miRNAs in different species. Meyers et al., (2008) further confirmed it in favor of plants miRNA annotation [32].

\subsection{Mimulus miRNAs Characterization}

According to MFOLD [26], the minimum folding free energies (MFE) of the newly identified Mimulus premiRNAs have a range from -12.3 to -81.2 with an average $-34.5 \mathrm{Kcal} \cdot \mathrm{mol}^{-1}$. The pre-miRNAs length ranges from 75 - $435 \mathrm{nt}$ with an average of $132 \mathrm{nt}$. The mature miRNA sequences length ranges from 20 - 23 nt. Majority (79.4\%) of the Mimulus miRNAs have 21 nt length, followed by $20 \mathrm{nt}$ (11.8\%), $22 \mathrm{nt}$ (5.9\%) and $23 \mathrm{nt}$ (3.0\%). The maximum (50.0\%) Mimulus miRNAs are observed having 2 mismatches with their homologs, followed by 1 (20.6\%), 3 (14.7\%), 4 (8.8\%) and 0 (5.9\%) mismatches. Majority (61.8\%) of Mimulus miRNAs is located on the 5' and remaining (38.2\%) are on the opposite 3' arms of the pre-miRNAs as illustrated in Figures 1(a), 1(b). The predicted miRNA stem-loop structures show that there are at least 12 - 21 nucleotides engaged in Watson-crick or G/U base pairings between the mature miRNA and the opposite arms (miRNAs*) in the stem region and the hairpin precursors do not contain large internal loops or bulges. The Mimulus miRNAs characterization such as source miRNAs, pre-miRNAs 

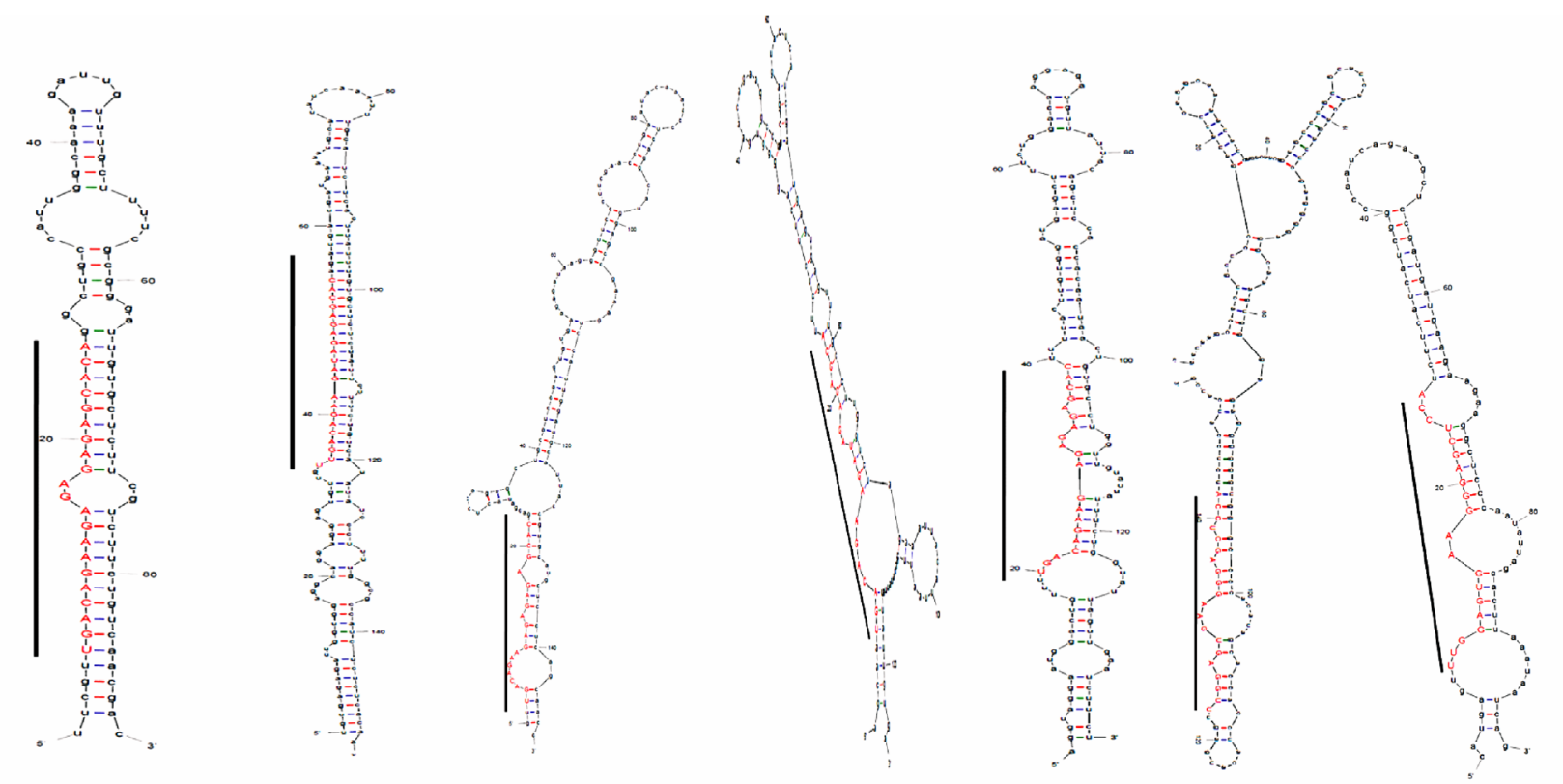

mle-MIR 156a

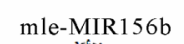

mle-MIR $156 \mathrm{c}$

mgu-MIR 157b

mgu-MIR157c mgu-MIR159b

mgu-MIR159c
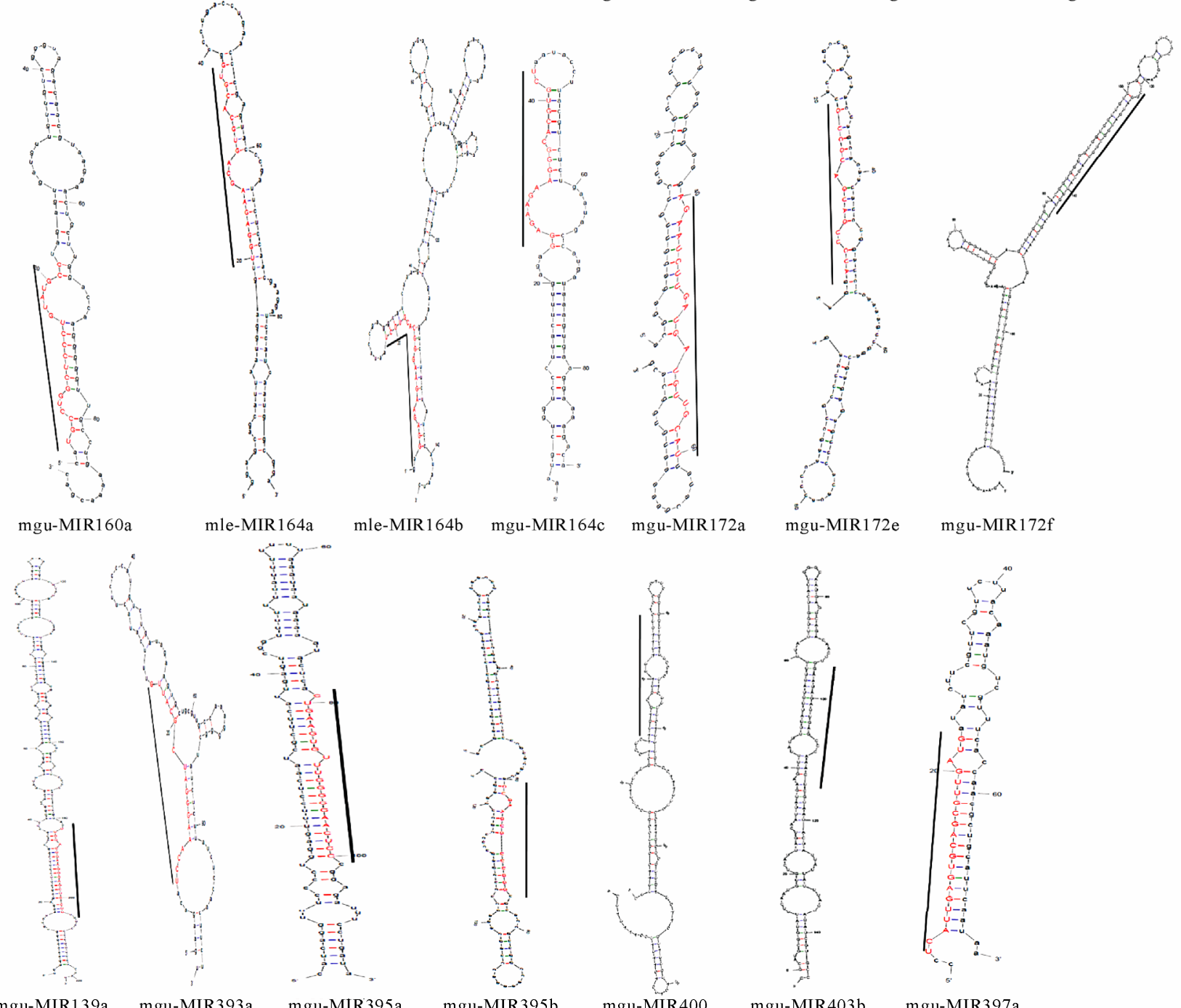

mgu-MIR172e

mgu-MIR $172 \mathrm{f}$

mgu-MIR139a

mgu-MIR395

mgu-MIR395b
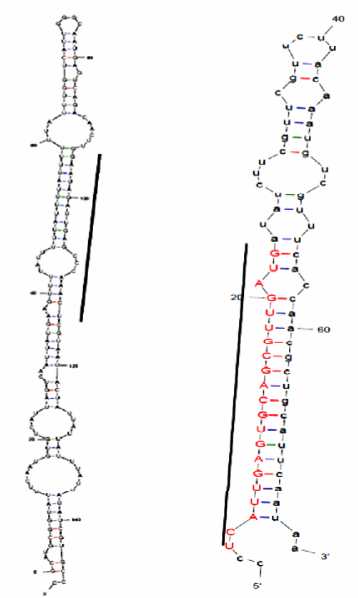

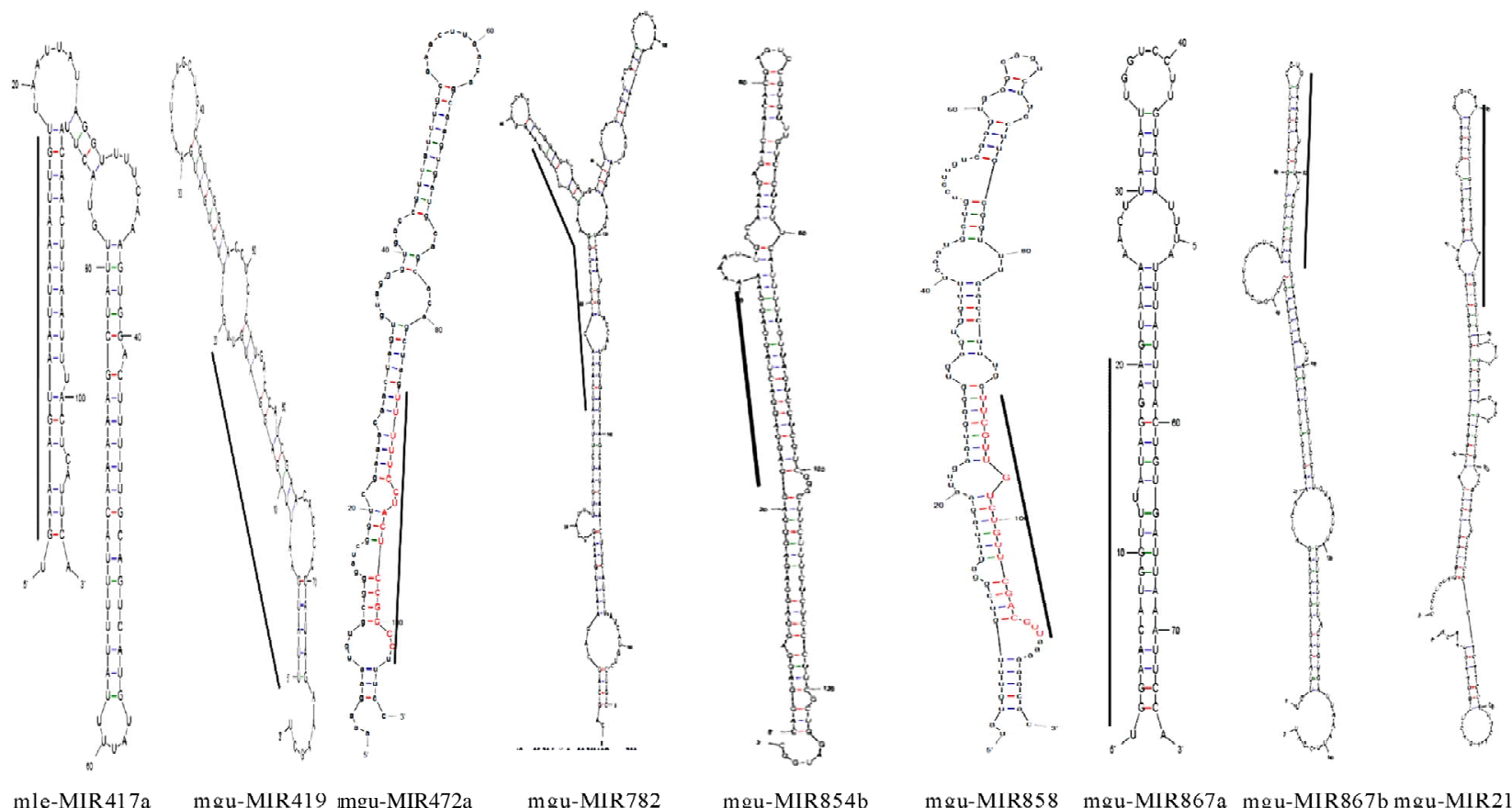

mle-MIR417a mgu-MIR419 mgu-MIR472a

mgu-MIR782

mgu-MIR854b

mgu-MIR858 mgu-MIR867a mgu-MIR867b mgu-MIR2112

(a)

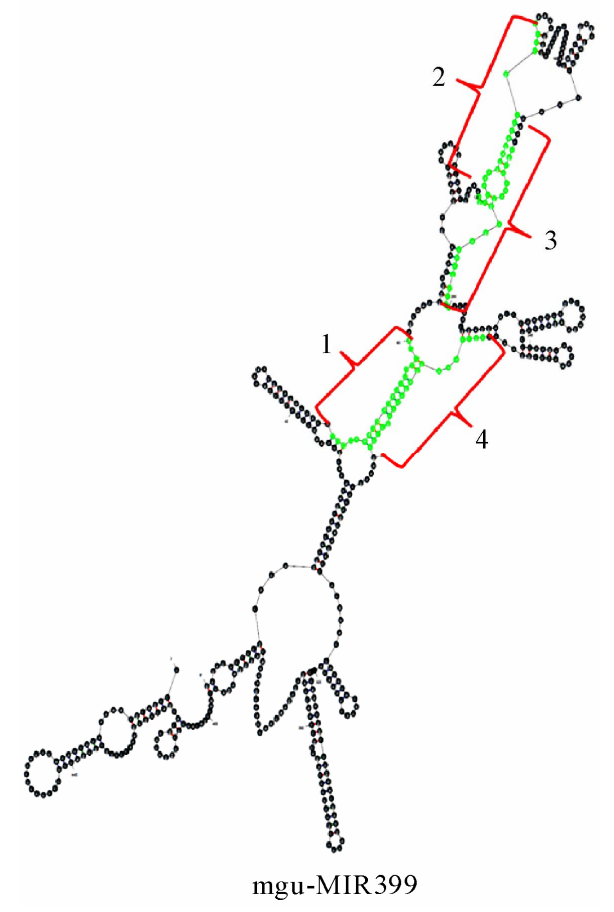

(b)

Figure 1. (a) Mimulus pre-miRNA secondary structures predicted with the help of Mfold. These structures are showing the mature miRNAs in stem portion, highlighted by a parallel line; (b) secondary structure of Mimulus pre-miRNA cluster (399). It is showing 4 mature miRNAs (highlighted by braces) in stem portion of a single pre-miRNA.

length (PL), minimum free folding energies (MFE), mature miRNA sequences (MS), number of mismatches (NM),mature sequence length (ML), source ESTs (SE), mature sequence arm (MSA) and GC percentage are summarized in Table 1. These findings are similar to the reported works of various groups working on miRNAs 
Table 1. Mimulus miRNAs' characterization. The novel identified Mimulus miRNAs are characterized in terms of PL $=$ Precursor miRNA Length, MFE = Minimum Free Energy, MS = Mature Sequence, MSA = Mature Sequence Arm, ML = Mature sequence Length, NM = Number of Mismatches (represented in bold \& enlarged font size) and SE = Source EST.

\begin{tabular}{|c|c|c|c|c|c|c|c|c|}
\hline $\begin{array}{l}\text { Mimulus } \\
\text { miRNAs }\end{array}$ & Ref. miRNAs & PL & MFE & MS & MSA & ML & NM & SE \\
\hline mle-MIR156a & ath-MIR156a & 90 & -50.50 & UGACAGAAGAGAGAGAGCAC & 5 & 20 & 1 & GR192132.1 \\
\hline mle-MIR156b & ath-MIR156a & 151 & -71.00 & UGACAGAAGAUAGAGAGCAC & 5 & 20 & 1 & GR194964.1 \\
\hline mle-MIR156c & ath-MIR156a & 147 & -40.80 & UGACAGAAGAGAGAGAGCAC & 5 & 20 & 1 & GR196312.1 \\
\hline mgu-MIR157b & ath-MIR157b & 124 & -26.50 & -UGACAGAAGAUAGAGAGCA- & 5 & 21 & 2 & GR180707.1 \\
\hline mgu-MIR157c & ath-MIR157b & 140 & -33.00 & UUGACAGAAGAGAGAGAGCAC & 5 & 21 & 1 & GR086652.1 \\
\hline mgu-MIR159b & ath-MIR159b & 154 & -45.40 & UUUGGAGUGAAGGGAGCUC-- & 3 & 21 & 2 & GO989625.1 \\
\hline mgu-MIR159c & ath-MIR159b & 100 & -36.70 & UUUGGAGUGAAGGGAGCUC-- & 5 & 21 & 2 & GO953516.1 \\
\hline mgu-MIR160a & ath-MIR160a & 92 & -27.80 & UGCCUGGCUCCCUGUAUGCC- & 5 & 21 & 1 & GR127528.1 \\
\hline mle-MIR164a & ath-MIR164a & 94 & -27.60 & UGGAGAAGCAGUGCACGUG-- & 5 & 21 & 3 & GR192152.1 \\
\hline mle-MIR164b & ath-MIR164a & 145 & -33.80 & -GGAGAAGAAGGGCACGUGCU & 5 & 21 & 2 & GR163353.1 \\
\hline mgu-MIR164c & ath-MIR164c & 90 & -21.70 & -GGAGAAGAAGGGCACGUGCU & 5 & 21 & 3 & GR156361.1 \\
\hline mgu-MIR172a & ath-MIR172a & 80 & -15.60 & AGAAUCUUGAUGAUGUUGCAU & 3 & 21 & 1 & GR008048.1 \\
\hline mgu-MIR172e & ath-MIR172e & 95 & -20.00 & ---AUCUUGAUGAUGCUGUAU & 5 & 21 & 4 & GR093896.1 \\
\hline mgu-MIR172f & ath-MIR172e & 180 & -42.90 & GGAAUCCUGAUGAUGCUGCAG & 3 & 21 & 2 & DV208903.1 \\
\hline mgu-MIR319a & ath-MIR319a & 221 & -78.70 & UUGGAGUGAAGGGAGCUCCA & 3 & 20 & 2 & GO989626.1 \\
\hline mgu-MIR393a & ath-MIR393a & 95 & -19.10 & AUCCAAAGGGAUCGCAUUG---- & 5 & 23 & 4 & GR132380.1 \\
\hline mgu-MIR395a & ath-MIR395a & 116 & -54.70 & CUGAAGUGUUUGGGGGAACUC & 3 & 21 & 0 & GR140768.1 \\
\hline mgu-MIR395b & ath-MIR395a & 125 & -26.5 & -UGAAGUGUUUGGGUGAACUC & 3 & 21 & 2 & GR127885.1 \\
\hline mgu-MIR397a & ath-MIR397a & 75 & -30.50 & UCAUUGAGUGCAGCGUUGAUG & 5 & 21 & 0 & GR112787.1 \\
\hline mgu-MIR399f & ath-MIR399f & 435 & -81.16 & $\begin{array}{l}\text { UGCCAAAGGAGAUUUGCCCAA } \\
\text { UGCCAAAGGAGAUUUGCCCAA } \\
\text { UGCCAAAGGAGAUUUGCCCAA } \\
\text { UGCCAAAGGAGAUUUGCCUGA }\end{array}$ & $\begin{array}{l}5 \\
5 \\
3 \\
3\end{array}$ & $\begin{array}{l}21 \\
21 \\
21 \\
21\end{array}$ & $\begin{array}{l}2 \\
2 \\
2 \\
3\end{array}$ & GR190746.1 \\
\hline mgu-MIR400 & ath-MIR400 & 140 & -20.20 & -AUGAGCGUAUUAUAAGUCAC & 5 & 21 & 2 & GR132977.1 \\
\hline mgu-MIR403b & ath-MIR403b & 147 & -25.90 & AUAGAUUGA-GCCCAAACUCG & 3 & 21 & 2 & GO970218.1 \\
\hline mle-MIR417 & ath-MIR417 & 108 & -14.90 & GAAAGUAAUUAAAUUGUU--A & 5 & 21 & 4 & GR203461.1 \\
\hline mgu-MIR419 & ath-MIR419 & 80 & -12.30 & UUAUGAAUU-UGAGGAUGUUG & 5 & 21 & 2 & GR038066.1 \\
\hline mgu-MIR472 & ath-MIR472 & 107 & -28.30 & UUUUUCCUACUCCGCCCUUUCC & 3 & 22 & 2 & GO976090.1 \\
\hline mgu-MIR782 & ath-MIR782 & 167 & -26.70 & -CAAACACCUUGGAAGCUUCUU & 5 & 22 & 3 & GR065602.1 \\
\hline mgu-MIR854 & ath-MIR854 & 130 & -51.3 & GAGGAGGAGAGGGAGGAGGAG & 5 & 21 & 2 & GR036365.1 \\
\hline mgu-MIR858 & ath-MIR858 & 119 & -27.20 & CUUCGUUGUCUGUUCGACCUU & 3 & 21 & 1 & GR102844.1 \\
\hline mgu-MIR867a & ath-MIR867 & 75 & -13.90 & UGGAACAUGGUUUA-UAGGAA & 5 & 21 & 2 & GR189481.1 \\
\hline mgu-MIR867B & ath-MIR867 & 145 & -26.10 & UGGAACAUGGUUUA-UAGGAA & 3 & 21 & 2 & GR148892.1 \\
\hline mgu-MIR2112 & ath-MIR2112 & 140 & -37.70 & CGCAAAUCUCGAUAUCAAUGU & 3 & 21 & 3 & GR150629.1 \\
\hline
\end{tabular}




\section{[21,22,33-35].}

Sometimes the miRNAs are expressed in clusters. These miRNAs are expressed either as pre-miRNAs clusters or non-precursor miRNAs clusters. The miRNA clusters are rarely observed in plants. In the current study we also identified the Mimulus miRNA 399 as pre-miRNA cluster (Figure 1(b). The Mimulus 399 pre-miRNA cluster was observed with four mature miRNA sequences. The same family is reported as cluster miRNA in plants [34].

To validate these novel miRNAs as strong candidates of miRNAs the relationship between them and known protein is very significant. The Mimulus pre-miRNAs were subjected through Blastx against the protein database at National Center for Biotechnology Information (NCBI) and found no homology with known proteins. This result is confirmed our identified pre-miRNAs as strong candidates in Mimulus.

\subsection{Sequence and Structural Features Filter}

The sequence and structural features filter is introduced by Li et al., (2006) in animals' miRNA validation and by Barozai et al., (2008) in plant [21,27]. It is useful to filter the false positive and validate the candidates. The filter is composed of four indices, namely GC content, core minimum free energy (mfe), hairpin mfe and the ratio of core mfe to hairpin mfe (ch_ratio).

As presented in (Table 2), identified miRNAs of Mimulus have a range of GC content (33.8 to 55.3), core mfe ( -56.10 to $\left.-19.80 \mathrm{kcal} \cdot \mathrm{mol}^{-1}, 72 \%\right)$, hairpin $\mathrm{mfe}$ ( -81 to $\left.-25 \mathrm{kcal} \cdot \mathrm{mol}^{-1}, 75 \%\right)$ and ch_ratio (40.0 to 95.0 , $80 \%)$. The GC content and ch_ratio are within the range given by Li et al. (2006) and Barozai et al. (2008) [21, 27].

\subsection{Conservation and Phylogenetic Studies of Mimulus miRNAs}

The novel Mimulus miRNA (mir-156) is studied for conservation and phylogeny. The Mimulus miRNA (mir156) has showed conservation with Arabidopsis thaliana, Vitis vinifera, Oryza sativa and Populus trichocarpa miRNAs as shown in Figure 2. The Phylogenetic analysis of the same miRNA (mir-156) sequences suggested that the Mimulus is more closed to Vitis vinifera (vvi) than the Populus trichocarpa (ptc) Arabidopsis thaliana (ath) and Oryza sativa (osa) as shown in Figure 3. The results are in agreement with the reported works [21, 34].

\subsection{Mimulus miRNA Targets}

The prediction of novel Mimulus miRNAs targets is a crucial step for validation of miRNAs identified on homology basis. Total 22 targets (Table 3) were annotated for the novel identified Mimulus miRNAs. Almost all of the predicted targets are reported as miRNA targeted proteins in various organisms [21,22,34].

The transcription factors are the famous and well known class of proteins targeted by miRNAs in almost all plant and animal species [4,20-22]. The novel identified Mimulus miRNAs also target this class of proteins. The predicted putative Mimulus targets for miRNAs; 156, 157, 164, 319, 397, 399, 417, 472, 782 and 854 are Squamosa-promoter binding protein, MYB transcripttional regulator, MYB transcription factor MIXTA-like 5 protein (MYBML5), MYB transcription factor MIXTA-like 6 protein (MYBML6), MYB transcription factor MIXTA-like 9 protein (MYBML9), MYB transcription factor MIXTA-like 5 protein (MYBML5), MYB transcriptional regulator, MYB transcription factor MIXTA- like 2 protein (MYBML2) and MYB transcription factor MIXTA-like 1 (MYBML1) respectively. The other Mimulus miRNA families' putative targets are Dihydrofla-vonol-4-reductase, Cycloidea-like protein A (CYCA), DNA-directed RNA polymerase II, NADH dehydrogenase, External transcribed spacer, Maturase (matR), Leafy-like protein and Maturase $\mathrm{K}$ (matK). Our findings are in agreement with the earlier reported works of many researchers group in the same field [10,20-22].

\section{CONCLUSIONS}

We have identified novel thirty four miRNAs belonging to twenty one (21) families in Mimulus from ESTs sequences. All twenty one (21) families and their thirty four members are repotted for the first time. These findings will be helpful in understanding the gene regulation

Table 2. Comparison of Mimulus, Arabidopsis thaliana and Li et al., Reference values of GC-content, core mfe, hairpin mfe and ch-ratio.

\begin{tabular}{ccccc}
\hline Reference & GC content & core $\mathbf{~ m f e}$ & Hairpin mfe & ch_ratio \\
\hline Li et al. & $30-60(93 \%)$ & $-42 \sim-17(99 \%)$ & $-50.2 \sim-24.2(99 \%)$ & $50-96(99 \%)$ \\
Arabidopsis Homologs & $36.4-51.1$ & $-54.2 \sim-23.5$ & $-79.4 \sim-48.3$ & $42-93$ \\
Mimulus & $33.8-55.3$ & $-56.1 \sim-19.8$ & $-81 \sim-25$ & $40-95$ \\
\hline
\end{tabular}


Table 3. Putative Mimulus miRNA targets. The Mimulus miRNA families and their putative targeted proteins function, Genbank Acc. and RNA-hybrid results are provided.

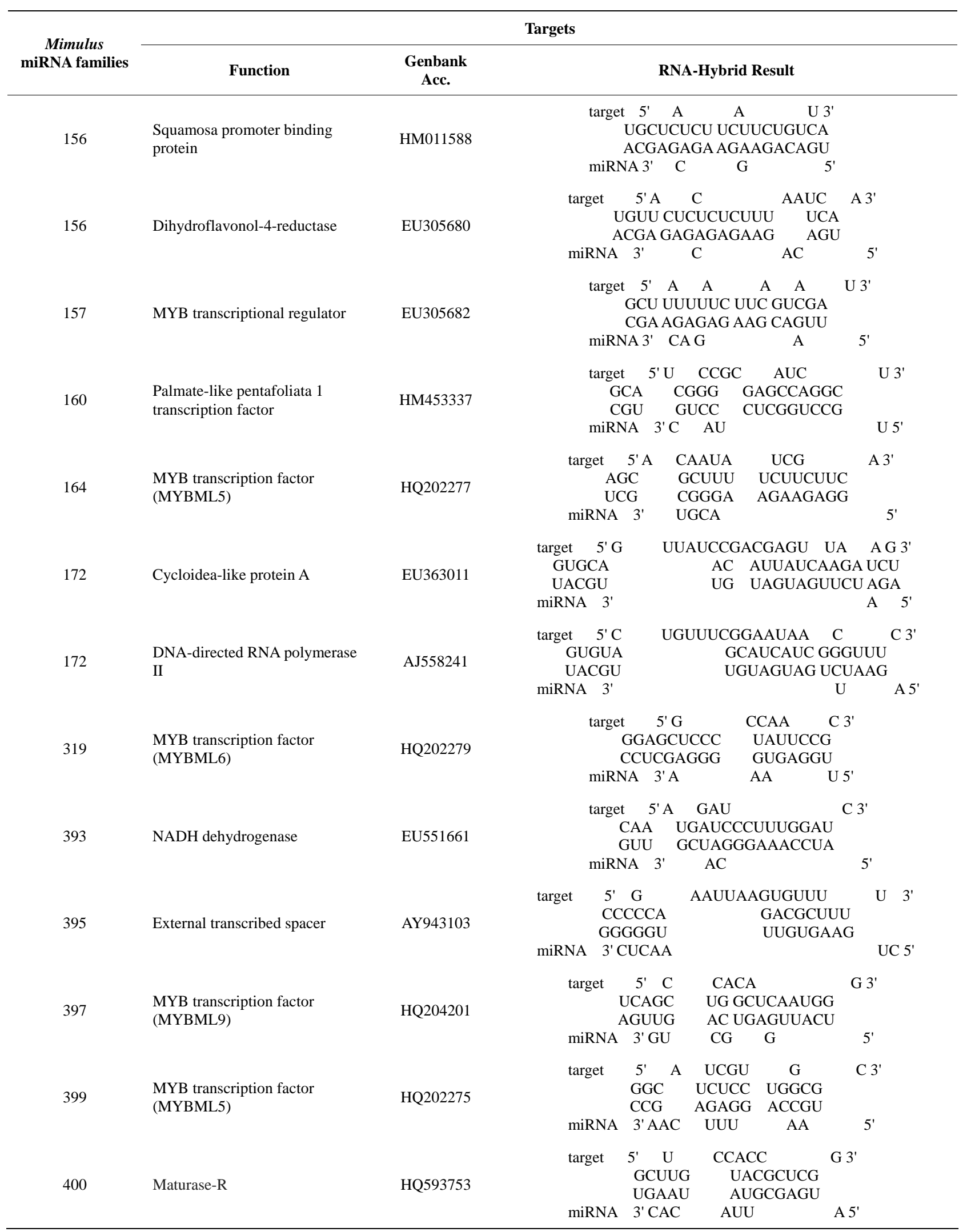




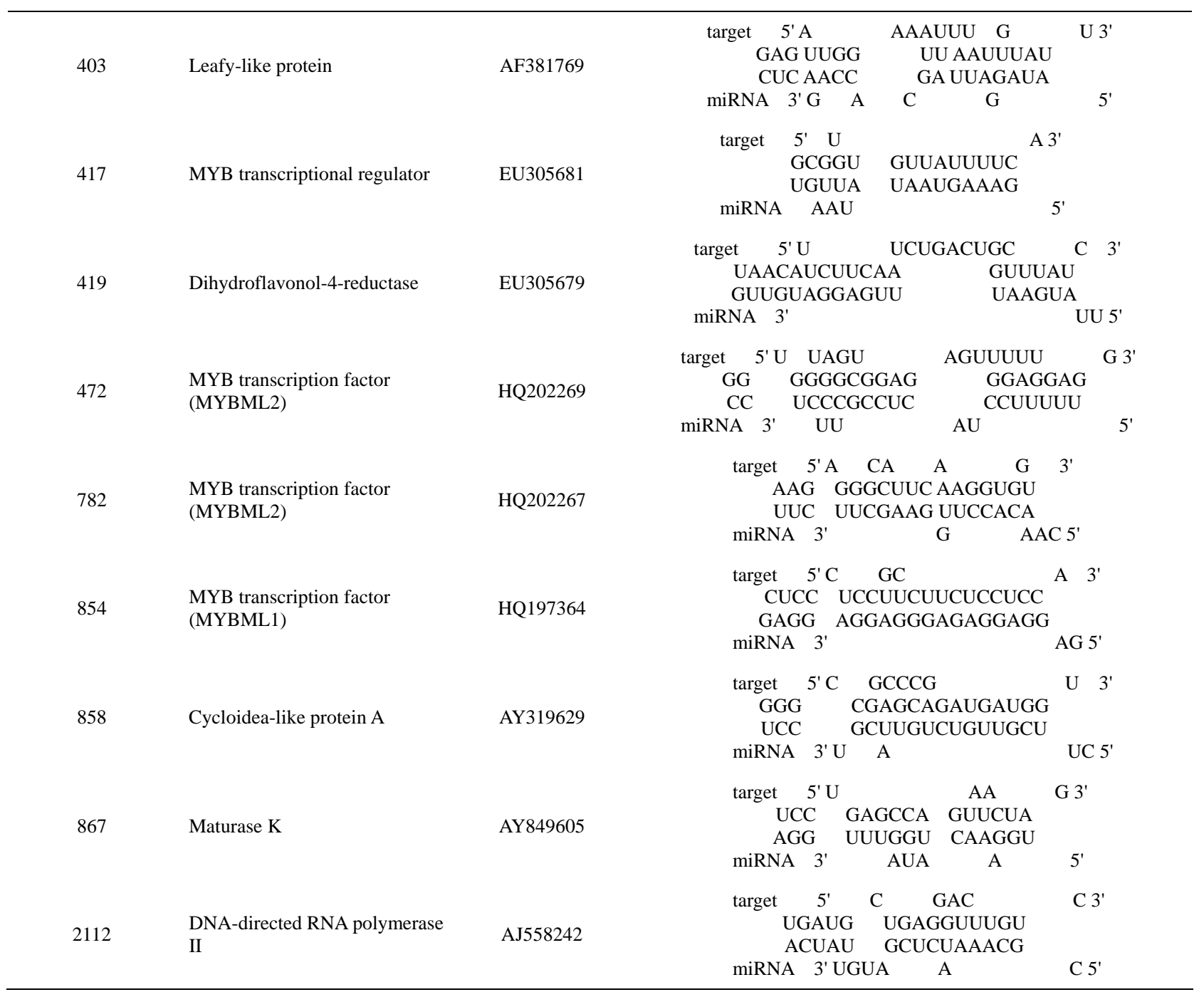

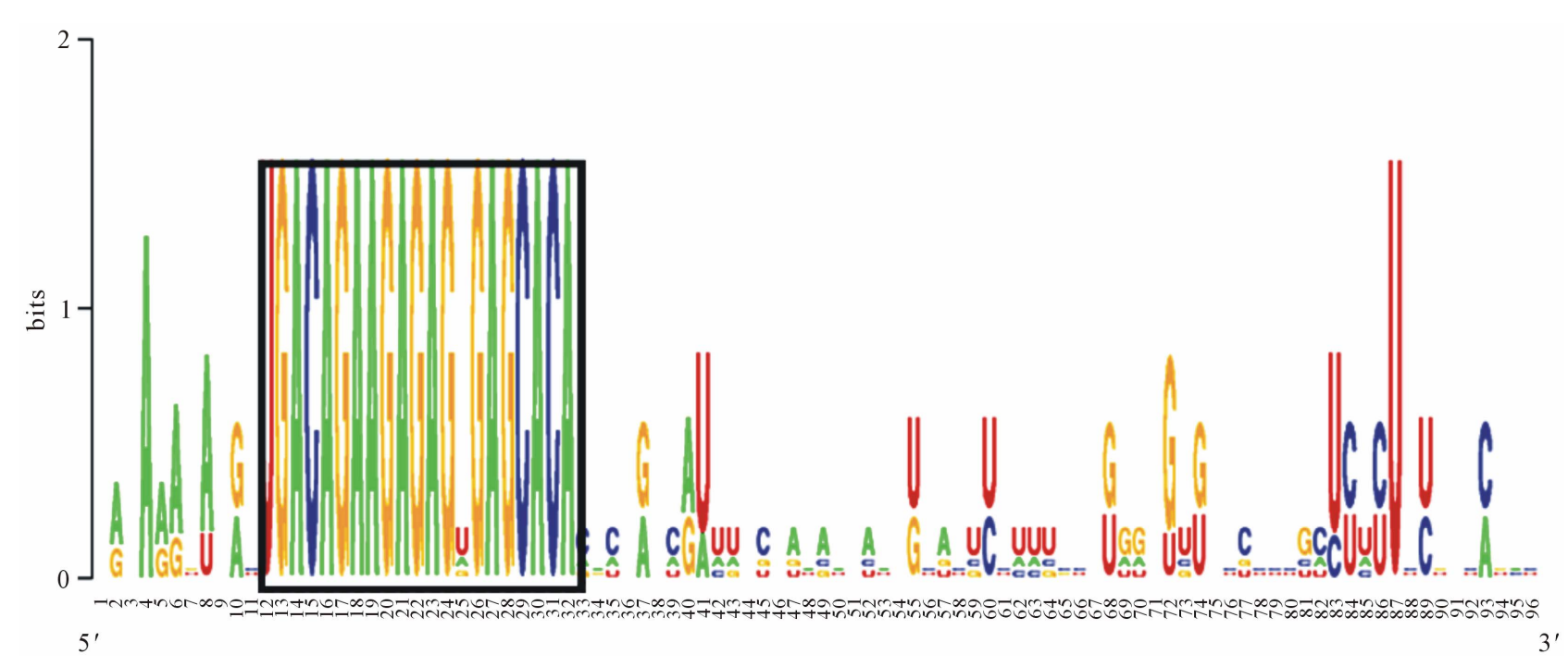

Figure 2. The Mimulus miRNA conservation studies. Alignment of pre-miRNA (156) of Mimulus with Arabidopsis thaliana, Vitis vinifera, Oryza sativa and Populus trichocarpa pre-miRNAs, using Weblogo: a sequence logo generator, showing miRNA sequences conservation, highlighted in a box. 


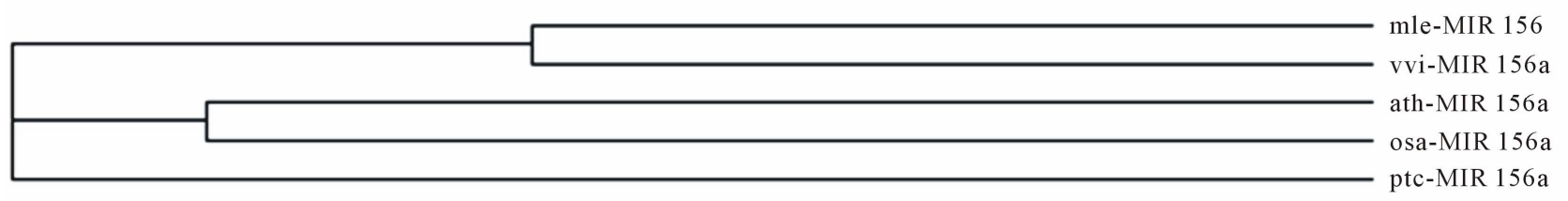

Figure 3. The Mimulus miRNA phylogenetic analysis. The Phylogenetic analysis of the pre-miRNA (156) of Mimulus (mle) with Arabidopsis thaliana (ath), Vitis vinifera (vvi), Oryza sativa (osa) and Populus trichocarpa (ptc) miRNAs, is done with the help of Clustalw and cladogram tree was generated using neighbor joining clustering method. The Phylogenetic tree showed that on the basis of pre-miRNA sequences, the Mimulus is more closed to Vitis vinifera (vvi) than the Populus trichocarpa (ptc) Arabidopsis thaliana (ath) and Oryza sativa (osa).

concept in the ecological model plant Mimulus. It also strengthens the bioinformatics approach for new premiRNAs identification from plant species whose genome is not yet sequenced. The ESTs based identification confirmed the miRNAs expression.

\section{REFERENCES}

[1] Beardsley, P.M. and Olmstead, R.G. (2002) Redefining Phrymaceae: The placement of Mimulus, tribe Mimuleae, and Phryma. American Journal of Botany, 89, 1093-1102. doi:10.3732/ajb.89.7.1093

[2] Fishman, L.A., Kelly, J., Morgan, E. and Willis, J.H. (2001) A genetic map in the Mimulus guttatus species complex reveals transmission ratio distortion due to heterospecific interactions. Genetics, 159, 1701-1716.

[3] Mica, E., Gianfranceschi, L. and Pe, M.E. (2006) Characterization of five microRNA families in maize. Journal of Experimental Botany, 57, 2601-2612. doi:10.1093/jxb/erl013

[4] Bonnet, E., Wuyts, J., Rouze, P. and Van-de-Peer, Y. (2004) Detection of 91 potential conserved plant microRNAs in Arabidopsis thaliana and Oryza sativa identifies important target genes. Proceedings of the National Academy of Sciences of the United States of America, 101, 11511-11516. doi:10.1073/pnas.0404025101

[5] Weber, M.J. (2005) New human and mouse microRNA genes found by homology search. FEBS Journal, 272, 59-73. doi:10.1111/j.1432-1033.2004.04389.x

[6] Bartel, D.P. (2004) MicroRNAs: Genomics, biogenesis, mechanism, and function. Cell, 116, 281-297. doi:10.1016/S0092-8674(04)00045-5

[7] Carrington, J.C. and Ambros, V. (2003) Role of microRNAs in plant and animal development. Science, 301, 336-338. doi:10.1126/science.1085242

[8] Hammond, S.C., Bernstein, E., Beach, D. and Hannon, G.J. (2000) An RNA-directed nuclease mediates posttranscriptional gene silencing in Drosophila cells. Nature, 404, 293-296. doi:10.1038/35005107

[9] Kurihara, Y. and Watanabe, Y. (2004) Arabidopsis micro-RNA biogenesis through dicer-like 1 protein functions. Proceedings of the National Academy of Sciences of the United States of America, 101, 12753-12758. doi:10.1073/pnas.0403115101

[10] Aukerman, M.J. and Sakai, H. (2003) Regulation of flowering time and floral organ identity by a microRNA and its APETALA2-Like target genes. Plant Cell, 15,
2730- 2741. doi:10.1105/tpc.016238

[11] Tang, G., Reinhart, B.J., Bartel, D.P. and Zamore, P.D. (2003) A biochemical framework for RNA silencing in plants. Genes \& Development, 17, 49-63. doi:10.1101/gad.1048103

[12] Chen, X. (2003) A microRNA as a translational repressor of APETALA2 in Arabidopsis flower development. Science, 303, 2022-2025. doi:10.1126/science.1088060

[13] Kidner, C.A. and Martienssen, R.A. (2005) The developmental role of microRNA in plants. Current Opinion in Plant Biology, 8, 38-44. doi:10.1016/j.pbi.2004.11.008

[14] Allen, E., Xie, Z., Gustafson, A.M. and Carrington, J.C. (2005) MicroRNA-directed phasing during transacting siRNA biogenesis in plants. Cell, 121, 207-221. doi:10.1016/j.cell.2005.04.004

[15] Yoshikawa, M., Peragine, A., Park, M.Y. and Poethig, R.S. (2005) A pathway for the biogenesis of transacting siRNAs in Arabidopsis. Genes \& Development, 19, 21642175. doi:10.1101/gad.1352605

[16] Lu, S., Sun, Y.H., Shi, R., Clark, C., Li, L. and Chiang, V.L. (2005) Novel and mechanical stress responsive microRNAs in Populus trichocarpa that are absent from Arabidopsis. The Plant Cell, 17, 2186-2203. doi:10.1105/tpc.105.033456

[17] Sunkar, R. and Zhu, J.K. (2004) Novel and stress-regulated microRNAs and other small RNAs from Arabidopsi. The Plant Cell, 16, 2001-2019. doi:10.1105/tpc.104.022830

[18] Johnson, S.M., Grosshansm, H., Shingara, J., Byrom, M. Jarvis, R., Cheng, A., Labourier, E., Reinert, K.L., Brown, D. and Slack, F.J. (2005) RAS is regulated by the let-7 microRNA family. Cell, 120, 635-647. doi:10.1016/j.cell.2005.01.014

[19] Bennasser, Y., Le, S.Y., Yeung, M.L. and Jeang, K.T. (2004) HIV-1 encoded candidate micro-RNAs and their cellular targets. Retroviro, 1, 43. doi:10.1186/1742-4690-1-43

[20] Frazier, T.P, Xie, F., Freistaedter, A., Burklew, C.E., Zhang, B. (2010) Identification and characterization of microRNAs and their target genes in tobacco (Nicotiana tabacum). Planta, 232, 1289-1308. doi:10.1007/s00425-010-1255-1

[21] Barozai, M.Y.K., Irfan, M., Yousaf, R., Ali, I., Qaisar, U., Maqbool, A., Zahoor, M., Rashid, B., Hussnain, T. and Riazuddin, S. (2008) Identification of micro-RNAs in cotton. Plant Physiology and Biochemistry, 46, 739-751. doi:10.1016/j.plaphy.2008.05.009

[22] Xie, F, Frazier, T.P. and Zhang, B. (2010) Identification 
and characterization of microRNAs and their targets in the bioenergy plant switchgrass (Panicum virgatum). Planta, 232, 417-434. doi:10.1007/s00425-010-1182-1

[23] Griffiths-Jones, S. (2004) The microRNA Registry. Nucleic Acids Research, 32D, 109-111. doi:10.1093/nar/gkh023

[24] Altschul, S.F., Gish, W., Miller, W., Myers, E.W. and Lipman, D.J. (1990) Basic local alignment search tool. Journal of Molecular Biology, 215, 403-410.

[25] Stephen, F.A., Madden, T.L., Schaffer, A.A., Zhang, J., Zhang, Z., Miller, W. and Lipman, D.J. (1997) Gapped BLAST and PSI-BLAST, a new generation of protein database search programs. Nucleic Acids Research, 25, 3389-3402. doi:10.1093/nar/25.17.3389

[26] Zuker, M. (2003) Mfold web server for nucleic acid folding and hybridization prediction. Nucleic Acids Research, 31, 3406-3415. doi:10.1093/nar/gkg595

[27] Li, S.C., Pan, C.U. and Lin, W.C. (2006) Bioinformatic discovery of microRNA precursors from human ESTs and introns. BMC Genomics, 7, 164. doi:10.1186/1471-2164-7-164

[28] Crooks, G.E., Hon, G., Chandonia, J.M. and Brenner, S.E. (2004) WebLogo: A sequence logo generator. Genome Research, 14, 1188-1190. doi:10.1101/gr.849004

[29] Larkin, M.A., Blackshields, G., Brown, N.P., Chenna, R., McGettigan, P.A., McWilliam, H., Valentin, F., Wallace,
I.M., Wilm, A., Lopez, R., Thompson, J.D., Gibson, T.J. and Higgins, D.G. (2007) ClustalW and ClustalX version 2. Bioinformatics, 23, 2947-2948. doi:10.1093/bioinformatics/btm404

[30] Kruger, J. and Rehmsmeier, M. (2006) RNAhybrid: MicroRNA target prediction easy, fast and flexible. Nucleic Acids Research, 34, Supplement 2, W451-W454.

[31] Ambros, V., Bartel, B. and Bartel, D.P. (2003) A uniform system for microRNA annotation. RNA, 9, 277-279. doi:10.1261/rna.2183803

[32] Meyers, B.C., Axtell, M.J., Bartel, B., et al. (2008) Criteria for annotation of plant MicroRNAs. The Plant Cell, 20, 3186-3190. doi:10.1105/tpc.108.064311

[33] Reinhart, B.J., Weinstein, E.G., Rhoades, M.W., Bartel, B. and Bartel, D.P. (2002) MicroRNAs in plants. Genes \& Development, 16, 1616-1626. doi:10.1101/gad.1004402

[34] Barozai, M.Y.K., Baloch, I.A. and Din, M. (2011) Computational identification of MicroRNAs and their targets in two species of evergreen Spruce tree (Picea). Waset, 75, 413-418.

[35] Barozai, M.Y.K., Baloch, I.A. and Din, M. (2011) Identification of MicroRNAs and their targets in Helianthus. Molecular Biology Reports. doi:10.1007/s11033-011-1004-y 